Document downloaded from:

http://hdl.handle.net/10251/148096

This paper must be cited as:

Calatayud-Gregori, J.; Cortés, J.; Jornet-Sanz, M. (2019). Random differential equations with discrete delay. Stochastic Analysis and Applications. 37(5):699-707. https://doi.org/10.1080/07362994.2019.1608833

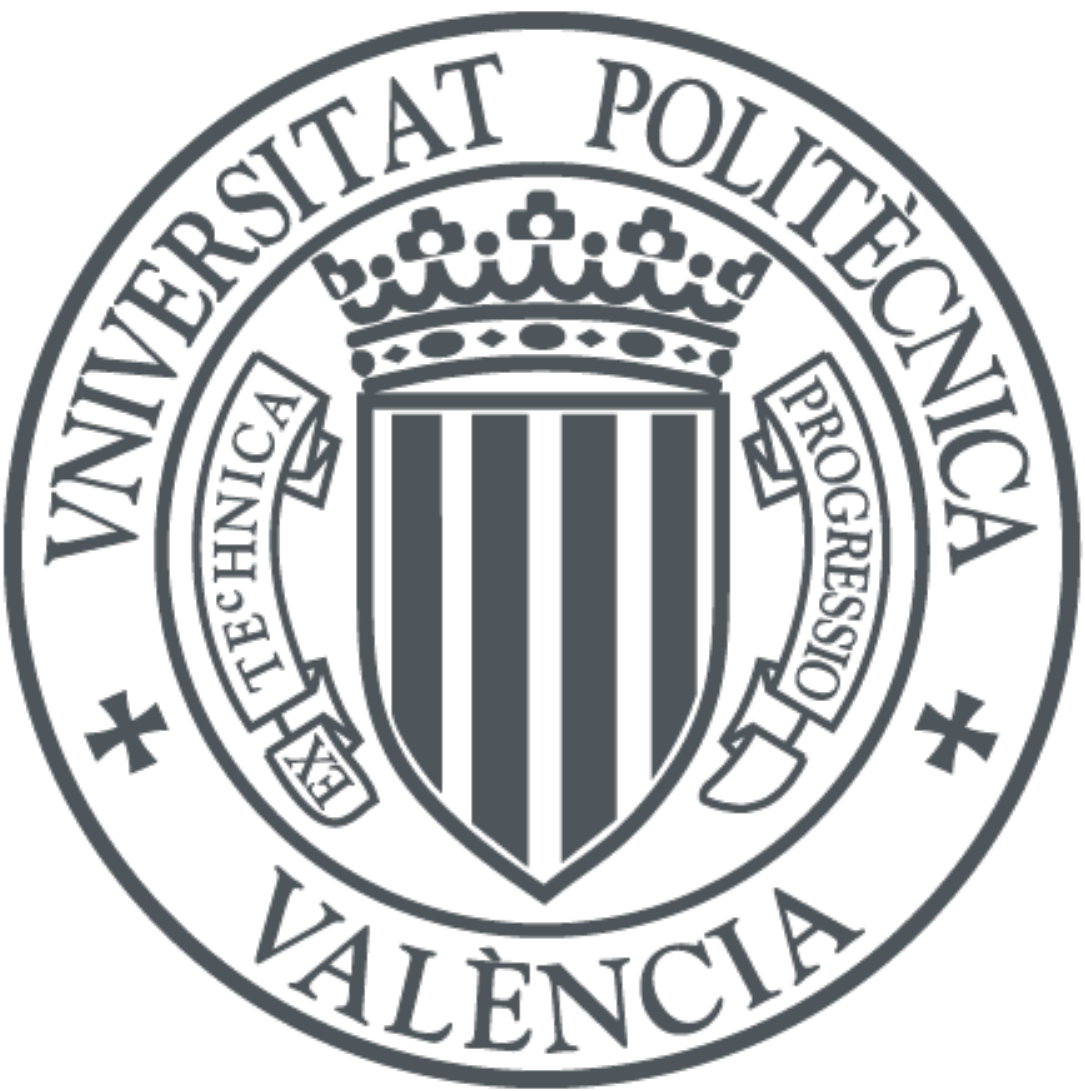

The final publication is available at

https://doi.org/10.1080/07362994.2019.1608833

Copyright Taylor \& Francis

Additional Information 


\title{
RANDOM DIFFERENTIAL EQUATIONS WITH DISCRETE DELAY
}

\author{
J. CALATAYUD ${ }^{1}$, J.-C. CORTÉS ${ }^{1}$, M. JORNET ${ }^{1}$ \\ ${ }^{1}$ Instituto Universitario de Matemática Multidisciplinar, \\ Universitat Politècnica de València, \\ Camino de Vera s/n, 46022, Valencia, Spain. \\ emails: jucagre@doctor.upv.es; jccortes@imm.upv.es; marjorsa@doctor.upv.es
}

\begin{abstract}
In this article, we study random differential equations with discrete delay $\tau>0: x^{\prime}(t, \omega)=f(x(t, \omega), x(t-\tau, \omega), t, \omega), t \geq t_{0}$, with initial condition $x(t, \omega)=g(t, \omega), t \in\left[t_{0}-\tau, t_{0}\right]$. The uncertainty in the problem is reflected via the outcome $\omega$. The initial condition $g(t)$ is a stochastic process. The term $x(t)$ is a stochastic process that solves the random differential equation with delay in a probabilistic sense. In our case, we use the $\mathrm{L}^{p}$ random calculus approach. We extend the classical Picard theorem for deterministic ordinary differential equations to $\mathrm{L}^{p}$ calculus for random differential equations with delay, via Banach fixedpoint theorem. We also relate $\mathrm{L}^{p}$ solutions with sample-path solutions. Finally, we utilize the theoretical ideas to solve the random autonomous linear differential equation with discrete delay.
\end{abstract}

Keywords: random differential equation with discrete delay, stochastic process, $\mathrm{L}^{p}$ random calculus, Banach fixed-point theorem.

AMS Classification 2010: 34F05, 34K50, 60H10, 65C30.

\section{INTRODUCTION}

The main goal of this paper is to contribute to extend the fundamental theory of deterministic differential equations with discrete delay to the random scenario. On the one hand, differential equations with delay provide a powerful approach to properly include memory information or aftereffects when modeling phenomena whose future dynamics depends not only on their present state, but also on their history. This feature often happens in different important problems: in viscoelastic problems in rheology, in the spread of a disease in epidemiology, in the aggregation of human capital in economics, etc., see [1, 2], for example. On the other hand, this kind of problems involve some type of inherent complexity, like impurities in the materials in viscoelasticity, lack of knowledge in the transmission features embedded in the contagion coefficient in epidemiology, intricate human behavior in economy, etc., that usually do not permit setting the coefficients of differential equations with delay in a deterministic manner but probabilistic. These facts lead to the study of differential equations with delay that consider randomness in their formulation. The way in which uncertainty (noise) is considered in the formulation of differential equations with delay has a profound impact in the mathematical 
tools required to solve the corresponding stochastic/random equations. When uncertainty is considered via a stochastic process having irregular sample behavior (e.g., nowhere differentiable), these equations are referred to as Stochastic Differential Equations with Delay (SDED). In general, the probabilistic behavior of the stochastic process governing the noise in SDED restricts itself to specific patterns, like for instance the Gaussian distribution when uncertainty is considered via the Wiener process. The analysis of this type of equations is based on a combination of Itô calculus and the machinery of equations with aftereffects [3]. Some recent interesting applications in engineering and epidemiology of SDED can be found in [4 -6], for example. There exists another way to consider uncertainty in the formulation of differential equation with delay that consists in directly assuming that the inputs (coefficients, forcing term, initial conditions, etc.) are random variables and/or stochastic processes whose sample behavior is regular (e.g. continuity), but allowing for a wider type of probability distributions like Binomial, Poisson, Beta, Gamma, Gaussian, etc. This type of equations are termed random differential equations with delay (RDED). This latter fact provides greater flexibility and makes very attractive RDED in dealing with real applications, since the parameters of the corresponding mathematical model must be quantified (adjusted) in order to properly describe the phenomena under study.

Although the analysis of both SDED and RDED is currently a topic under development, there are still many important deterministic results for differential equations with delay that have not been formulated in the stochastic/random context yet. Regarding SDED, we must say that some contributions have already considered SDE with different types of delay, such as SDE with infinite delay, SDE with random delay and SDE of impulsive type with randomly varying trial lengths, for example [7 9$]$. However, the study of RDED needs further analysis. Indeed, while the theory and applications for random ordinary differential equations have been extensively studied in the extant literature 10 13, there is still a lack of analysis for important variations of RDE, like the ones formulated via delays. We must point out that one exception is the generalization to the random context of the classical theory of fractional differential equations performed in 14, 15. In the same spirit that this latter contribution, the aim of this contribution is to advance in the generalization of some relevant results for RDED using the theory of $\mathrm{L}^{p}$ random Lebesgue spaces [16] and the so-called sample-path approach [10, Appendix A], [17]. Specifically, in Section 2, we extend the classical Picard theorem for deterministic differential equations for RDED via the Banach fixed-point theorem by taking advantage of $\mathrm{L}^{p}$ random calculus. This result allows us to establish the relationship between $\mathrm{L}^{p}$ and sample-path solutions. In Section 3, we apply the results established in Section 2 to solve the autonomous linear RDED in the $\mathrm{L}^{p}$ sense.

\section{MAIN RESUlts ON RANDOM DIFFERENTIAL EQUATIONS WITH DELAY}

We consider the general form of a random differential equation problem with discrete delay $\tau>0$ :

$$
\left\{\begin{array}{l}
x^{\prime}(t, \omega)=f(x(t, \omega), x(t-\tau, \omega), t, \omega), \quad t \in I=\left[t_{0}, T\right] \\
x(t, \omega)=g(t, \omega), \quad t_{0}-\tau \leq t \leq t_{0} .
\end{array}\right.
$$


The uncertainty is introduced into (2.1) via an outcome $\omega$ that belongs to the sample space $\Omega$ of an underlying complete probability space $(\Omega, \mathcal{F}, \mathbb{P})$, where $\mathcal{F} \subseteq 2^{\Omega}$ is the $\sigma$-algebra of events and $\mathbb{P}$ is the probability measure. This outcome $\omega$ may be omitted, and we may simply write $x(t), g(t)$ and $f(x(t), x(t-\tau), t)$ instead. The terms $g(t)$ and $x(t)$ are stochastic process (the initial condition and the solution, respectively), from $\Omega$ to $\mathbb{R}^{n}, n \geq 1$. In vector form, we write them as $x(t)=$ $\left(x_{1}(t), \ldots, x_{n}(t)\right)$ and $g(t)=\left(g_{1}(t), \ldots, g_{n}(t)\right)$.

The solution stochastic process $x(t)$ will be considered in the Lebesgue space $\mathrm{L}_{n}^{p}$ $(1 \leq p \leq \infty)$, which is defined as the set of random vectors $y=\left(y_{1}, \ldots, y_{n}\right): \Omega \rightarrow \mathbb{R}^{n}$ with finite norm

$$
\|y\|_{p}=\max _{1 \leq j \leq n}\left\|y_{j}\right\|_{\mathrm{L}^{p}}
$$

where $\left\|y_{j}\right\|_{\mathrm{L}^{p}}=\left(\mathbb{E}\left[\left|y_{j}\right|^{p}\right]\right)^{1 / p}$ is the norm of the Lebesgue space $\mathrm{L}^{p}$, being $\mathbb{E}$ the expectation operator. This space $\mathrm{L}_{n}^{p}$ has the structure of a Banach space [10]. In the case $p=2$, the operational calculus and the corresponding convergence are usually referred to as mean square calculus and mean square convergence, respectively.

In the Banach space $\mathrm{L}_{n}^{p}$, one can define $\mathrm{L}^{p}$ continuity, differentiability and Riemann integrability, by taking each limit from the classical definition in the topology of $\mathrm{L}_{n}^{p}$. See $[10,18$ for a presentation on this random calculus.

A pre-established hypothesis in our setting is that the initial condition process $g:\left[t_{0}-\tau, t_{0}\right] \rightarrow \mathrm{L}_{n}^{p}$ is continuous.

The map $f$ is regarded as follows: $f=\left(f_{1}, \ldots, f_{n}\right): \mathrm{L}_{n}^{p} \times \mathrm{L}_{n}^{p} \times I \rightarrow \mathrm{L}_{n}^{p}$. A pre-established assumption is that $f$ is continuous, in the sense that if $\left\{t_{m}\right\}_{m=1}^{\infty}$ is a sequence of times that tends to $t$ in $I$ and $\left\{x_{m}\right\}_{m=1}^{\infty}$ and $\left\{y_{m}\right\}_{m=1}^{\infty}$ are two sequences of stochastic processes that tend to $x$ and $y$ in $\mathrm{L}_{n}^{p}$, respectively, then $\left\{f\left(x_{m}, y_{m}, t_{m}\right)\right\}_{m=1}^{\infty}$ tends to $f(x, y, t)$ in $\mathrm{L}_{n}^{p}$.

We say that the stochastic process $x(t)=\left(x_{1}(t), \ldots, x_{n}(t)\right)$ is an $\mathrm{L}^{p}$ solution to (2.1) on $I$, if $x: I \rightarrow \mathrm{L}_{n}^{p}$ is $\mathrm{L}^{p}$ differentiable on $I, \mathrm{~L}^{p}$ continuous on $\left[t_{0}-\tau, t_{0}\right] \cup I$, $x^{\prime}(t)=f(x(t), x(t-\tau), t)$ for all $t \in I$, and $x(t)=g(t)$ for all $t \in\left[t_{0}-\tau, t_{0}\right]$.

Notice that being a solution to (2.1) is conserved by equivalence of stochastic processes (recall that two stochastic processes $y(t)$ and $z(t)$ are equivalent if, for each $t$, the relationship $y(t)=z(t)$ holds almost surely on $\Omega$ ). Thus, $\mathrm{L}^{p}$ solutions to (2.1) are actually equivalence classes. The equivalence class of a stochastic process $x$ will be denoted as $\hat{x}$. This is a distinctive feature compared with sample-path solutions [10, App. A] and [17].

At a first step, we relate (2.1) with its associated integral form:

Proposition 2.1. Let $x: I \rightarrow \mathrm{L}_{n}^{p}$. We have that $x$ is a solution to 2.1 in the $\mathrm{L}^{p}$ sense, if and only if

- $x$ is continuous on $\left[t_{0}-\tau, T\right]$;

- $x(t)=g\left(t_{0}\right)+\left(\mathrm{L}^{p}\right) \int_{t_{0}}^{t} f(x(s), x(s-\tau), s) \mathrm{d} s$, for each $t \in I$ (here, the integral stands for the $\mathrm{L}^{p}$ Riemann integral);

- $x(t)=g(t)$, for each $t \in\left[t_{0}-\tau, t_{0}\right]$.

Proof. Suppose that $x$ is an $\mathrm{L}^{p}$ solution to (2.1). The first and third conditions are satisfied by definition of $\mathrm{L}^{p}$ solution. Let us check the second condition. We have $x^{\prime}(t)=f(x(t), x(t-\tau), t)$ on $I$ in $\mathrm{L}^{p}$. As both $x$ and $f$ are continuous, the process 
$f(x(\cdot), x(\cdot-\tau), \cdot)$ is $\mathrm{L}^{p}$-continuous: indeed, if $\left\{t_{m}\right\}_{m=1}^{\infty}$ is a sequence in $I$ that tends to $t \in I$, then $x\left(t_{m}\right) \rightarrow x(t)$ and $x\left(t_{m}-\tau\right) \rightarrow x(t-\tau)$ in $\mathrm{L}_{n}^{p}$ as $m \rightarrow \infty$, by $\mathrm{L}^{p}$ continuity of $x$ on $\left[t_{0}-\tau, T\right]$, so $f\left(x\left(t_{m}\right), x\left(t_{m}-\tau\right), t_{m}\right) \rightarrow f(x(t), x(t-\tau), t)$ in $\mathrm{L}_{n}^{p}$ as $m \rightarrow \infty$, by continuity of $f$. Hence, $x^{\prime}$ is $\mathrm{L}^{p}$-continuous. By [10, p. $\left.101(1)\right], x^{\prime}$ is $\mathrm{L}^{p}$ Riemann integrable on $I$, and by the Fundamental Theorem of $\mathrm{L}^{p}$ calculus [10, p. 104 $(6)], x(t)=g\left(t_{0}\right)+\left(\mathrm{L}^{p}\right) \int_{t_{0}}^{t} x^{\prime}(s) \mathrm{d} s=g\left(t_{0}\right)+\left(\mathrm{L}^{p}\right) \int_{t_{0}}^{t} f(x(s), x(s-\tau), s) \mathrm{d} s$.

On the contrary, suppose the three conditions. We want to demonstrate that $x$ is a solution to (2.1) in the $\mathrm{L}^{p}$ sense. Since $x$ and $f$ are continuous, the process $f(x(\cdot), x(\cdot-\tau), \cdot)$ is $\mathrm{L}^{p}$-continuous. By $[10$, p. $101(1)], f(x(\cdot), x(\cdot-\tau), \cdot)$ is $\mathrm{L}^{p}$ Riemann integrable on $I$, so that writing $\left(\mathrm{L}^{p}\right) \int_{t_{0}}^{t} f(x(s), x(s-\tau), s) \mathrm{d} s$ makes sense. By [10, p. $103(5)], x$ is $\mathrm{L}^{p}$ differentiable on $I$ with $x^{\prime}(t)=f(x(t), x(t-\tau), t)$.

In what follows, we extend the classical Picard theorem for deterministic ordinary differential equations to $\mathrm{L}^{p}$ calculus for random differential equations with delay, via Banach fixed-point theorem.

Theorem 2.2. Suppose that $f$ satisfies the following Lipschitz condition:

$$
\|f(x, y, t)-f(u, v, t)\|_{p} \leq k(t) \max \left\{\|x-u\|_{p},\|y-v\|_{p}\right\},
$$

for $x, y \in \mathrm{L}_{n}^{p}, t \in I$, and certain $k \in \mathrm{L}^{1}(I)$. Then (2.1) has a unique $\mathrm{L}^{p}$ solution $x: I \rightarrow \mathrm{L}_{n}^{p}$.

Proof. Since $k \in \mathrm{L}^{1}(I)$, then $\lim _{t \rightarrow t_{0}^{+}} \int_{t_{0}}^{t} k(s) \mathrm{d} s=0$. This is a consequence of the Dominated Convergence Theorem: as $\lim _{t \rightarrow t_{0}^{+}} k(s) 1_{\left(t_{0}, t\right)}(s)=0$ for all $s \in I$ and $k(s) 1_{\left(t_{0}, t\right)}(s) \leq k(s) \in \mathrm{L}^{1}(I)$, we can interchange the limit and the integral. Thus, we can choose $\alpha>t_{0}$ such that, for all $t \in I_{\alpha}=\left[t_{0}, \alpha\right] \subseteq I, \int_{t_{0}}^{t} k(s) \mathrm{d} s \leq 1 / 2$.

Consider the vector space

$$
\mathcal{A}=\left\{x:\left[t_{0}-\tau, \alpha\right] \rightarrow \mathrm{L}_{n}^{p} \text { continuous, } x(t)=g(t) \text { on }\left[t_{0}-\tau, t_{0}\right]\right\},
$$

with the norm

$$
\|x\|_{\mathcal{A}}=\sup _{t \in\left[t_{0}-\tau, \alpha\right]}\|x(t)\|_{p}
$$

Notice that $\|x\|_{\mathcal{A}}$ is well-defined, because, by the $\mathrm{L}_{n}^{p}$ continuity of $x$, the real map $t \in\left[t_{0}-\tau, \alpha\right] \mapsto\|x(t)\|_{p}$ is continuous, therefore $\sup _{t \in\left[t_{0}-\tau, \alpha\right]}\|x(t)\|_{p}<\infty$.

Let us check that $\mathcal{A}$ is a Banach space. Let $\left\{x_{j}\right\}_{j=1}^{\infty}$ be a Cauchy sequence in $\mathcal{A}$. Let $\epsilon>0$. There exists $j_{0}(\epsilon)$ such that, for all $j \geq m \geq j_{0}(\epsilon),\left\|x_{j}-x_{m}\right\|_{\mathcal{A}}<\epsilon$. This implies that $\left\|x_{j}(t)-x_{m}(t)\right\|_{p}<\epsilon$, for each $t \in\left[t_{0}-\tau, \alpha\right]$. Thereby, $\left\{x_{j}(t)\right\}_{j=1}^{\infty}$ is Cauchy in $\mathrm{L}_{n}^{p}$, for each $t \in\left[t_{0}-\tau, \alpha\right]$, and by the completeness of $\mathrm{L}_{n}^{p}$, we can define the process $x(t)=\lim _{j \rightarrow \infty} x_{j}(t)$, for each $t \in\left[t_{0}-\tau, \alpha\right]$. Letting $j \rightarrow \infty$ in the inequality $\left\|x_{j}(t)-x_{m}(t)\right\|_{p}<\epsilon$, we obtain $\left\|x(t)-x_{m}(t)\right\|_{p} \leq \epsilon$, for all $m \geq j_{0}(\epsilon)$ and $t \in\left[t_{0}-\tau, \alpha\right]$. Taking supremum on $t$, we derive that $x \in \overline{\mathcal{A}}$ and $\left\|x-x_{m}\right\|_{\mathcal{A}} \leq \epsilon$, for all $m \geq j_{0}(\epsilon)$, so that $x_{m} \rightarrow x$ in $\mathcal{A}$.

Consider the map $\Lambda: \mathcal{A} \rightarrow \mathcal{A}$ such that

$$
\Lambda x(t)=\left\{\begin{array}{l}
g(0)+\left(\mathrm{L}^{p}\right) \int_{t_{0}}^{t} f(x(s), x(s-\tau), s) \mathrm{d} s, \quad t \in I_{\alpha}, \\
g(t), \quad t \in\left[t_{0}-\tau, t_{0}\right] .
\end{array}\right.
$$


If $x \in \mathcal{A}$, as both $x$ and $f$ are continuous, the process $f(x(\cdot), x(\cdot-\tau), \cdot)$ is $\mathrm{L}^{p}$ continuous. By [10, p. $103(5)], \Lambda x \in \mathcal{A}$, so $\Lambda$ is well-defined.

By Proposition 2.1, $x: I_{\alpha} \rightarrow \mathrm{L}_{n}^{p}$ is a solution to (2.1) if and only if $x \in \mathcal{A}$ and $\Lambda(x)=x$.

By Banach fixed-point theorem [19, Ch. 1], it suffices to check that $\Lambda$ is a contraction. Let $x, y \in \mathcal{A}$ and $t \in I_{\alpha}$ (if $t \in\left[t_{0}-\tau, t_{0}\right]$, we know that $\Lambda x(t)-\Lambda y(t)=$ $g(t)-g(t)=0)$. By $[10$, p. $102(3)]$,

$$
\begin{aligned}
\|\Lambda x(t)-\Lambda y(t)\|_{p} & =\left\|\left(\mathrm{L}^{p}\right) \int_{t_{0}}^{t}(f(x(s), x(s-\tau), s)-f(y(s), y(s-\tau), s)) \mathrm{d} s\right\|_{p} \\
& \leq \int_{t_{0}}^{t}\|f(x(s), x(s-\tau), s)-f(y(s), y(s-\tau), s)\|_{p} \mathrm{~d} s \\
& \leq \int_{t_{0}}^{t} k(s) \max \left\{\|x(s)-y(s)\|_{p},\|x(s-\tau)-y(s-\tau)\|_{p}\right\} \mathrm{d} s \\
& \leq \int_{t_{0}}^{t} k(s) \mathrm{d} s\|x-y\|_{\mathcal{A}} \leq \frac{1}{2}\|x-y\|_{\mathcal{A} .}
\end{aligned}
$$

Taking supremum on $t \in\left[t_{0}-\tau, \alpha\right],\|\Lambda x-\Lambda y\|_{\mathcal{A}} \leq \frac{1}{2}\|x-y\|_{\mathcal{A}}$, therefore $\Lambda$ is a contraction.

Thus, there exists a unique solution to (2.1) on $I_{\alpha}$. Let us extend this solution to $I$. Let $r_{0} \geq \alpha$ be the supremum of numbers $r$ in $\left(t_{0}, T\right]$ such that $x(t)$ is a solution to (2.1) on $\left[t_{0}, r\right]$. Suppose that $r_{0}<T$. If this supremum were attained, so that $x(t)$ is a solution to 2.1) on $\left[t_{0}, r_{0}\right]$, then we could find a local solution to

$$
\left\{\begin{array}{l}
y^{\prime}(t)=f(y(t), y(t-\tau), t), \quad t \geq r_{0}, \\
y(t)=x(t), \quad r_{0}-\tau \leq t \leq r_{0}
\end{array}\right.
$$

so that $x(t)$ would be extended to the right. Thus, the supremum is not attained and $x(t)$ is a solution on $\left[t_{0}, r_{0}\right)$. Pick $t_{1} \in\left(t_{0}, r_{0}\right)$ such that $\int_{t_{1}}^{r_{0}} k(s) \mathrm{d} s \leq 1 / 4$. Take $\beta \in\left(r_{0}, T\right)$ such that $\int_{r_{0}}^{\beta} k(s) \mathrm{d} s \leq 1 / 4$. Then $\int_{t_{1}}^{\beta} k(s) \mathrm{d} s \leq 1 / 2$. We may apply the above proof with $t_{1}$ instead of $t_{0}$ and $\beta$ instead of $\alpha$ to derive that there is a unique solution $z(t)$ to

$$
\left\{\begin{array}{l}
z^{\prime}(t)=f(z(t), z(t-\tau), t), \quad t_{1} \leq t \leq \beta \\
z(t)=x(t), \quad t_{1}-\tau \leq t \leq t_{1} .
\end{array}\right.
$$

Then we have extended $x(t)$ to the right (on $\left[r_{0}, \beta\right]$ ). This is a contradiction by definition of $r_{0}$, therefore $x(t)$ may be defined on the whole $I$.

To finish this section, we relate $\mathrm{L}^{p}$ solutions and sample-path solutions. For random differential equations with no delay, this study has been undertaken in [17]. We will relate (2.1) to a random differential equation problem with no delay via the method of steps, and by using some results from [17], we will prove that every equivalence class that solves (2.1) in an $\mathrm{L}^{p}$ sense has a product measurable version that solves 2.1) in the sample-path sense. Thus, candidates for $L^{p}$ solutions to (2.1) are merely its deterministic solutions randomized with an outcome $\omega$. 
Theorem 2.3. Suppose that the initial condition $g$ has continuous sample-paths on $\left[t_{0}-\tau, t_{0}\right]$. If $\hat{x}: I \rightarrow \mathrm{L}_{n}^{p}$ solves the $\mathrm{L}^{p}$ problem (2.1), then there exists an equivalent stochastic process $x: I \times \Omega \rightarrow \mathbb{R}^{n}$, product measurable, solving (2.1) in the sample-path sense.

Proof. On the interval $\left[t_{0}, t_{0}+\tau\right]$, our random delay differential equation problem (2.1) is equivalent to a random ordinary differential equation problem, by the method of steps: our equivalence class $\hat{x}$ satisfies

$$
\left\{\begin{array}{l}
\hat{x}^{\prime}(t)=f(\hat{x}(t), g(t-\tau), t), \quad t \in\left[t_{0}, t_{0}+\tau\right], \\
\hat{x}\left(t_{0}\right)=g\left(t_{0}\right) .
\end{array}\right.
$$

By [17, Th. 3 (a)], there is a version $x^{1}:\left[t_{0}, t_{0}+\tau\right] \times \Omega \rightarrow \mathbb{R}^{n}$, product measurable, that solves 2.2 in the sample-path sense.

On the interval $\left.\left[t_{0}+\tau, t_{0}+2 \tau\right], 2.1\right)$ becomes again a random ordinary differential equation problem: $\hat{x}$ satisfies

$$
\left\{\begin{array}{l}
\hat{x}^{\prime}(t)=f\left(\hat{x}(t), x^{1}(t-\tau), t\right), \quad t \in\left[t_{0}+\tau, t_{0}+2 \tau\right], \\
\hat{x}\left(t_{0}+\tau\right)=x^{1}\left(t_{0}+\tau\right)
\end{array}\right.
$$

By $\left[17\right.$, Th. 3 (a)], there exists a version $x^{2}:\left[t_{0}+\tau, t_{0}+2 \tau\right] \times \Omega \rightarrow \mathbb{R}^{n}$, product measurable, that solves 2.3 in the sample-path sense.

Continuing in this manner, we construct a version of $\hat{x}$ which is a sample-path solution $x^{i}:\left[t_{0}+(i-1) \tau, t_{0}+i \tau\right] \times \Omega \rightarrow \mathbb{R}^{n}$, product measurable, to

$$
\left\{\begin{array}{l}
\hat{x}^{\prime}(t)=f\left(\hat{x}(t), x^{i-1}(t-\tau), t\right), \quad t \in\left[t_{0}+(i-1) \tau, t_{0}+i \tau\right] \\
\hat{x}\left(t_{0}+(i-1) \tau\right)=x^{i-1}\left(t_{0}+(i-1) \tau\right)
\end{array}\right.
$$

We define the stochastic process

$$
x(t)= \begin{cases}g(t), & t \in\left[t_{0}-\tau, t_{0}\right], \\ x^{1}(t), & t \in\left[t_{0}, t_{0}+\tau\right], \\ x^{2}(t), & t \in\left[t_{0}+\tau, t_{0}+2 \tau\right], \\ \vdots & \\ x^{i}(t), & t \in\left[t_{0}+(i-1) \tau, t_{0}+i \tau\right], \\ \vdots & \end{cases}
$$

This stochastic process belongs to the equivalence class $\hat{x}$ and solves (2.1) in the sample-path sense on its whole domain $I$.

Indeed, notice that $x(t)$ is well-defined because of the initial condition of $(2.4)$. It belongs to the equivalence class $\hat{x}$ because, at each subinterval of the form $\left[t_{0}+(i-\right.$ 1) $\left.\tau, t_{0}+i \tau\right], x^{i}$ is a version of $\hat{x}$. On the other hand, as $g$ is sample-path continuous on $\left[t_{0}-\tau, t_{0}\right]$ and each $x^{i}$ is sample-path continuous on $\left[t_{0}+(i-1) \tau, t_{0}+i \tau\right]$, we derive that $x(t)$ has continuous sample-paths on $\left[t_{0}-\tau, T\right]$. Finally, for the differentiability of the sample-paths of $x$ on $I$, if $t_{1}$ is an interior point of any of the subintervals of the form $\left(t_{0}+(i-1) \tau, t_{0}+i \tau\right)$, then there exists $x^{\prime}\left(t_{1}\right)=\left(x^{i}\right)^{\prime}\left(t_{1}\right)=f\left(x^{i}\left(t_{1}\right), x^{i-1}\left(t_{1}-\tau\right), t_{1}\right)=$ $f\left(x\left(t_{1}\right), x\left(t_{1}-\tau\right), t_{1}\right)$, by (2.4). If $t_{1}=t_{0}+(i-1) \tau$, then we check the equality of the left and right derivatives of the sample-paths of $x: x_{-}^{\prime}\left(t_{0}+(i-1) \tau\right)=$ 
$\left(x^{i-1}\right)^{\prime}\left(t_{0}+(i-1) \tau\right)=f\left(x^{i-1}\left(t_{0}+(i-1) \tau\right), x^{i-1}\left(t_{0}+(i-2) \tau\right), t\right)=\left(x^{i}\right)^{\prime}\left(t_{0}+(i-1) \tau\right)=$ $x_{+}^{\prime}\left(t_{0}+(i-1) \tau\right)$, so there exists $x^{\prime}\left(t_{0}+(i-1) \tau\right)=f\left(x\left(t_{0}+(i-1) \tau\right), x\left(t_{0}+(i-2) \tau\right), t\right)$. This shows that the sample-paths of $x$ solve (2.1) on $I$.

\section{Solving the RANDOM AUtonomous lineAr DifFERENTIAL EQUATION WITH DISCRETE DELAY}

The autonomous linear differential equation with discrete time delay $\tau>0$ is given by

$$
\left\{\begin{array}{l}
x^{\prime}(t)=a x(t)+b x(t-\tau), \quad t \geq 0 \\
x(t)=g(t), \quad-\tau \leq t \leq 0,
\end{array}\right.
$$

where $a$ is the coefficient of the non-delay component, $b$ is the parameter of the delay term, and the function $g(t)$ defined on $[-\tau, 0]$ is the initial condition. The method of steps provides the solution to the deterministic problem (3.1), expressed in terms of the delayed exponential function, see [20]: if $g \in C^{1}([-\tau, 0])$, then

$$
x(t)=\mathrm{e}^{a(t+\tau)} \mathrm{e}_{\tau}^{b_{1}, t} g(-\tau)+\int_{-\tau}^{0} \mathrm{e}^{a(t-s)} \mathrm{e}_{\tau}^{b_{1}, t-\tau-s}\left(g^{\prime}(s)-a g(s)\right) \mathrm{d} s,
$$

where $b_{1}=\mathrm{e}^{-a \tau} b$,

$$
\mathrm{e}_{\tau}^{c, t}= \begin{cases}0, & -\infty<t<-\tau, \\ 1, & -\tau \leq t<0, \\ 1+c \frac{t}{1 !}, & 0 \leq t<\tau, \\ 1+c \frac{t}{1 !}+c^{2} \frac{(t-\tau)^{2}}{2 !}, & \tau \leq t<2 \tau, \\ \vdots & \vdots \\ \sum_{k=0}^{n} c^{k} \frac{(t-(k-1) \tau)^{k}}{k !}, & (n-1) \tau \leq t<n \tau,\end{cases}
$$

is the delayed exponential function, $c, t \in \mathbb{R}$, and $n=\lfloor t / \tau\rfloor+1$ (here $\lfloor\cdot\rfloor$ denotes the integer part).

The randomization of (3.1) consists in assuming that the system depends on an outcome $\omega$ of an experiment:

$$
\left\{\begin{array}{l}
x^{\prime}(t, \omega)=a(\omega) x(t, \omega)+b(\omega) x(t-\tau, \omega), \quad t \geq 0 \\
x(t, \omega)=g(t, \omega), \quad-\tau \leq t \leq 0
\end{array}\right.
$$

The parameters $a=a(\omega)$ and $b=b(\omega)$ are random variables, and $g(t)=g(t, \omega)$ is a stochastic process, all of them defined in an underlying complete probability space $(\Omega, \mathcal{F}, \mathbb{P})$. The term $x(t)=x(t, \omega)$ is the solution stochastic process in some probabilistic sense.

The formal solution to 3.3 is obtained after randomization of 3.2 :

$x(t, \omega)=\mathrm{e}^{a(\omega)(t+\tau)} \mathrm{e}_{\tau}^{b_{1}(\omega), t} g(-\tau, \omega)+\int_{-\tau}^{0} \mathrm{e}^{a(\omega)(t-s)} \mathrm{e}_{\tau}^{b_{1}(\omega), t-\tau-s}\left(g^{\prime}(s, \omega)-a(\omega) g(s, \omega)\right) \mathrm{d} s$,

where $b_{1}(\omega)=\mathrm{e}^{-a(\omega) \tau} b(\omega)$ and the integral is understood in the sample-path sense. The stochastic process (3.4) is a solution to (3.3) in the sample-path sense, under the assumption that the sample-paths of $g$ belong to $C^{1}([-\tau, 0])$. 
We use the theory from Section 2 to prove that (3.4) is a rigorous $\mathrm{L}^{p}$ solution to (3.3), under certain suppositions on $a, b$ and $g(t)$.

Theorem 3.1. If $a$ and $b$ are bounded random variables, and $g(t)$ has $C^{1}([-\tau, 0])$ sample-paths, then (3.4) is the unique $\mathrm{L}^{p}$ solution to (3.3) on $[0, \infty)$.

Proof. The map $f: \mathrm{L}^{p} \times \mathrm{L}^{p} \times[0, \infty) \rightarrow \mathrm{L}^{p}$ is defined as follows: $f(x, y, t)=a x+b y$. For $a$ and $b$ bounded, we have that $\|f(x, y, t)\|_{p}=\|a x+b y\|_{p} \leq\|a\|_{\infty}\|x\|_{p}+$ $\|b\|_{\infty}\|y\|_{p}<\infty$, so $f$ is well-defined. For the Lipschitz condition, we have that $\|f(x, y, t)-f(u, v, t)\|_{p}=\|a(x-u)+b(y-v)\|_{p} \leq\|a\|_{\infty}\|x-u\|_{p}+\|b\|_{\infty}\|y-v\|_{p} \leq$ $2 \max \left\{\|a\|_{\infty},\|b\|_{\infty}\right\} \max \left\{\|x-u\|_{p},\|y-v\|_{p}\right\}$. We take $k(t)=2 \max \left\{\|a\|_{\infty},\|b\|_{\infty}\right\}$, which is integrable on any compact interval $[0, T], T>0$. Then Theorem 2.2 applies: problem (3.3) has a unique $\mathrm{L}^{p}$ solution on $[0, \infty)$, let us refer to it as an equivalence class $\hat{y}(t)$.

By Theorem 2.3, there exists an equivalent stochastic process $y(t)$, product measurable, solving (3.3) in the sample-path sense. But, since $g(t)$ has $C^{1}([-\tau, 0])$ sample-paths, we know that the unique sample-path solution is given by $x(t)$ defined as (3.4). Then $y$ and $x$ are indistinguishable stochastic processes. This implies that $x$ belongs to the equivalence class $\hat{y}$, so $x$ is an $\mathrm{L}^{p}$ solution to $(3.3)$ on $[0, \infty)$, as wanted.

This condition of boundedness for the random coefficients $a$ and $b$ may be restrictive, as we are not allowing common probability distributions such as Gamma, Poisson, or even Gaussian. In practice, there is the possibility of truncating the support of these unbounded probability distributions on a large interval. By using the generalized Markov's inequality, it can be demonstrated that any second-order random variable can be truncated on the interval [mean $\pm 10 \cdot$ deviation], so that this interval contains $99 \%$ of the mass/density probability regardless the probability distribution of the involved random variable.

From a theoretical point of view, as shown in [17, Example p. 541], the boundedness of the random coefficients is necessary: it was proved that, in order for a random autonomous and homogeneous linear differential equation of first-order (i.e., $\left.x^{\prime}(t)=a x(t)\right)$ to have an $\mathrm{L}^{p}$ solution for every initial condition $x(0)=x_{0}$ in $\mathrm{L}^{p}$, one needs the random coefficient $a$ to be bounded.

\section{ACKNOWLEDGEMENTS}

This work has been supported by the Spanish Ministerio de Economía y Competitividad grant MTM2017-89664-P. The author Marc Jornet acknowledges the doctorate scholarship granted by Programa de Ayudas de Investigación y Desarrollo (PAID), Universitat Politècnica de València.

\section{Conflict of Interest Statement}

The authors declare that there is no conflict of interests regarding the publication of this article. 


\section{REFERENCES}

[1] Erneux, T. (2009). Applied Delay Differential Equations. New York: Springer.

[2] Kuang, Y. (1993). Delay Differential Equations with Applications in Population Dynamics. San Diego, California, USA: Academic Press.

[3] Kolmanovskii, V. B., Shaikhet, L. (1997). Control of Systems with Aftereffect. New York: AMS, Translations of Mathematical Monographs.

[4] Fridman, E., Shaikhet, L. (2017). Stabilization by using artificial delays: an LMI approach. Automatica 81: 429-437. DOI: 10.1016/j.automatica.2017.04.015.

[5] Shaikhet, L., Korobeinikov, A. (2016). Stability of a stochastic model for HIV-1 dynamics within a host. Appl. Anal. 95(6): 1228-1238. DOI: 10.1080/00036811.2015.1058363.

[6] Caraballo, T., Colucci, R., Guerrini, L. (2018). On a predator prey model with nonlinear harvesting and distributed delay. Commun. Pure Appl. Ana. 17(6): 2703-2727. DOI: 10.3934/cpaa.2018128.

[7] Caraballo, T., Garrido-Atienza, M. J., Schmalfuss, B., Valero, J. (2017). Attractors for a random evolution equation with infinite memory: theoretical results. Discrete Cont. Dyn.-B. 22(5): 1779-1800. DOI: 10.3934/dcdsb.2017106.

[8] Krapivsky, P. L., Luck, J. M., Mallick, K. (2011). On stochastic differential equations with random delay. J. Stat. Mech.-Theory E. 2011. DOI: 10.1088/1742-5468/2011/10/P10008.

[9] Liu, S., Debbouche, A., Wang, J. R. (2017). On the iterative learning control for stochastic impulsive differential equations with randomly varying trial lengths. J. Comput. Appl. Math. 312: 47-57. DOI: 10.1016/j.cam.2015.10.028.

[10] Soong, T. T. (1973). Random Differential Equations in Science and Engineering. New York: Academic Press.

[11] Dorini, F. A., Cecconello, M. S., Dorini, L. B. (2016). On the logistic equation subject to uncertainties in the environmental carrying capacity and initial population density. Commun. Nonlinear Sci. 33: 160-173. DOI: 10.1016/j.cnsns.2015.09.009.

[12] Slama, H., El-Bedwhey, N. A., El-Depsy, A., Selim, M. M. (2017). Solution of the finite Milne problem in stochastic media with RVT Technique. Eur. Phys. J. Plus 132:505. DOI: 10.1140/epjp/i2017-11763-6.

[13] Nouri, K., Ranjbar, H., Torkzadeh, L. (2019). Modified stochastic theta methods by ODEs solvers for stochastic differential equations. Commun. Nonlinear Sci. 68: 336-346. DOI: 10.1016/j.cnsns.2018.08.013.

[14] Lupulescu, V., Ntouyas, K. N. (2012). Random fractional differential equations. International Electronic Journal of Pure and Applied Mathematics 4(2): 119-136.

[15] Lupulescu, V., O'Reagan, D., Rahman, G. U. (2014). Existence results for random fractional differential equations. Opuscula Mathematica 34(4): 813-825. DOI: 10.7494/OpMath.2014.34.4.813.

[16] Loève, M. (1977). Probability Theory. Vol. II. New York: Springer.

[17] Strand, J. L. (1970). Random ordinary differential equations. J. Differ. Equations 7(3): 538553. DOI: 10.1016/0022-0396(70)90100-2.

[18] Villafuerte, L., Braumann, C. A., Cortés, J. C., Jódar, L. (2010). Random differential operational calculus: theory and applications. Comput. Math. Appl. 59(1): 115-125. DOI: 10.1016/j.camwa.2009.08.061.

[19] Granas, A., Dugundji, J. (2003). Fixed Point Theory. New York: Springer-Verlag.

[20] Khusainov, D. Y., Pokojovy, M., Azizbayov, E. (2014). Classical solvability for linear 1d heat equation with constant delay. Zhurnal Obchyslyuval'nï ta Prykladnö̈ Matematyky 115: 76-87. 